\title{
Update in Neuroimaging
}

\author{
Mohit Neema and Rohit Bakshi
}

\begin{abstract}
Department of Neurology, Brigham and Women's Hospital, Laboratory for Neuroimaging Research, Partners MS Center, Harvard Medical School, One Brookline Place, Suite 602, Brookline, Massachusetts 02445
\end{abstract}

Neuroimaging has emerged as an invaluable tool in the study of central nervous system (CNS) structure and function, shedding light on both structural and functional changes. A wide array of neuroimaging techniques, such as computerized tomography (CT), magnetic resonance imaging (MRI), and positron emission tomography (PET), have been part of an unprecedented technological revolution, empowering our ability to diagnose and monitor neurologic conditions. Neuroimaging has a particularly important role in the field of neurotherapeutics for several reasons. The first is its ability to provide early and accurate diagnosis of neurologic disorders so that the appropriate therapy can be started. The second is its ability to monitor the evolution of chronic neurologic disorders so that patients experiencing breakthrough disease, who might require a change in therapy, may be identified. The third is its ability to probe structural and functional changes in both animal and human subjects, resulting in a better understanding of disease mechanisms and the identification of potential new therapeutic targets. We aim to present the current state and future prospects of neuroimaging techniques in evaluating neurological disorders with an emphasis on their impact on the field of neurotherapeutics.

We have included 11 review articles in this volume written by the world's leading authorities. To set the stage, the volume begins with a review of imaging of small animal models of CNS diseases, which is at the forefront in the translation of neuroimaging techniques from basic research to human studies. The subsequent reviews focus on human neuroimaging in major CNS disorders, including stroke, hemorrhage, trauma, multiple sclerosis, amyotrophic lateral sclerosis, Parkinson's disease, dementia, psychiatric disorders, and infections. Each article presents an overview of the role of the most relevant imaging modalities (e.g., CT, MRI, PET, angiography/interventional, and/or neurosonology). The reviews provide a critical analysis of the role of these techniques in clinical diagnosis, longitudinal monitoring, and neurotherapeutic evaluation/development. We conclude with a review of an emerging neuroimaging technique-optical coherence tomography, which has generated significant interest due to its ability to readily quantify damage to the retinal nerve fiber layer and, in turn, provide a surrogate of neurodegeneration, neuroprotection, and repair.

We would like to express our gratitude to the authors for the generous contribution of their time and expertise to this volume. Despite our wish to make this edition comprehensive, because of space limitations and publication deadlines, we have not adequately covered a number of neuroimaging-related topics, such as normal aging, epilepsy, neuro-oncology, and spinal cord disorders. Nonetheless, we hope that this volume will prove useful to the trainees, practitioners, and investigators in the clinical and imaging fields of the neurosciences and pique their interest in future studies that may expand and refine the applications of these exciting technologies.

Acknowledgments: We thank Ms. Sophie Tamm for her valuable administrative and editorial assistance on this entire project.

\footnotetext{
Address correspondence and reprint requests to: Mohit Neema Brigham \& Women's Hospital, Harvard Medical School, Laboratory for Neuroimaging Research, One Brookline Place, Suite 602, Brookline, MA 02445. E-mail: mneema@bwh.harvard.edu
} 